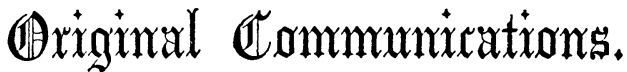

\section{ON INTRAUTERINE FRACTURES: WITH AN ILLUSTRATIVE CASE.}

By T. Herbert Barker, M.D., F.R.C.S., Bedford.

[Read before the South Midland Branch, May 21st, 185\%.]

[Concludca from page 809.$]$

Cause of Intracterine Fractures. It will have been renarked, that in the majority of the cases described in the preceding part of this paper, the fractured condition of the foetal bones was attributed to some lind of injury inflicted on the abdomen of the mother in the latter months of pregnancy. This observation, while it deserves to be remembered, does not claim such serious consideration as a cause of the accident to the child, as might at first sight be supposed. When, as practitioners of midwifery, we recall the many instances in which labour occurs after such accidents; when we consider what an enormous and often irregular pressure is exerted on the fotal bones with impunity during even natural labour; and when we further recollect the almost incredible force that may sometimes be employed in unnatural labour, without injury to the osseous framework of the infant, we must in a measure exclude the idea of fracture while the child is enclosed in the uterus and surrounded by the amniotic fluid. Yet that a blow on the abdomen may affect the child, has been argued for, on the ground that wounds of the skin of the fotus hare been met with, resulting apparently from a fall on the abdomen by the mother during the last weeks of pregnancy. In Dr. Iichardson's MS. Fothergillian Essay, already referred to, there is the history of a case reported to the Royal Medical and Chirurgical Society by Mr. Jones. The labour in this instance was easy: the pelvis was large. The mother had fallen down a few weeks before delivery. On the back of the foetus there existed an extensive wound, reaching from the third dorsal vertebra, across the scapula, and along the back of the humerus, to within an inch of the elbow. Part of this wound was cicatrised; the other half was granulating. Here the occurrence of the wound, antecedently to the birth of the child, was fully demonstrated; and its connexion with the fall, to which the mother had been subjected, was thus rendered probable.

Dr. R. U. West, of Alford, in writing to me on this subject, says he cannot at all see how the bones of the foetus can be fractured before birth by a fall of the mother. I confess myself to the same difficulty. Whatever force should convey to the fœtus such a blow as would fracture its bones, must be conveyed through the uterus itself, and should consequently injure that organ primarily; yet, in the cases described, we have the most marked absence of all evidence which would indicate any uterine injury whatever. It seems feasible, however, that in some few cases the fractures occurred after extreme violence to the mother. The cases recorded by Dr. Montgomery, and before referred to, aprear to have been of this kind.

Again, it must always be remembered that the bones are by natural composition less liable to fracture during fœetal life than at any other period. There is at this time less of earthy and more of organic matter. Hence, such bones are supple; they bend easily under the pressure incident to delivery ; they scarcely admit of being broken. With these facts before us, we must, therefore, be exceedingly cautious in supposing that an accident to the pregnant mother can alone give rise to fracture of the fotal bones. The accident of $a$ fall on the abdomen may be a coincidence; it may be a favouring circumstance; but it can never account for the injury in full.

Some instances have been noted in which certain peculiar injuries have been inflicted on the limbs of the child by the umbilical cord. Dr. Montgomery (op.cit.) and Professor Simpson attribute amputation of the limbs in utero, to the pressure exerted on the limbs either by the cord or by plastic bands.
In the case of fracture of a single limb with a band or a fold of the cord surrounding the fractured part, and giving evidence of pressure on the soft parts, it might be fair to attribute the division of bone to the local effect. But when many bones are broken, and the soft parts are entire, this view is excluded from the field of argument.

In speaking on the general question of fracture of the fœtal bones, Billard lays great stress on the statement, that a division of the long bones, which might easily be mistaken for fracture, may be in some cases nothing more than arrest of ossification of the ossifying cartilages. Dr. West, in his letter to me, suggests with great acuteness, that the case I have described was possibly of this character; and asks:-Are the fractures dependent on arrest of development and on the deficiency of animal matter at special points? The answer to this question suggests in part, and it may be altogether, the nature of the case before us.

On examining the bones of the fotus, to which I have especially drawn attention, it was plain that while there was an absence of development in the superficial cranial bones, there was nothing in the condition of the long bones to lead to that view of Billard, which would attribute the divisions met with to a mere arrest of ossification in the cartilages. We cannot look at this bone structure, as displayed in the specimens before us, without seeing distinctly that the fractures are complete, and that at the part where the breakage occurs, the ossific development, in the form of earthy material, is even more strongly marked out than in the extremities of the bone, which are indeed solely cartilaginous. We must then set down the predisposing cause of fracture as connected with an irregular as well as an imperfect development of the osseous structure.

Now, the question arises, what is the form of bone-disease here present? Is it what is commonly called rachitis? Is it what is known under the name of fragilitas ossium? The first named disorder is the one common to infancy, and depends upon a supposed deficiency of earthy matter; the second is a disease of adult life, and depends on a supposed increase of earthy material. I say supposed decrease and supposed increased proportion of earthy materials; becausc these specific variations in the constitution of bone, as marking specific forms of disease, are based on most uncertain data. Un these points I would direct the attention of the society to Mr. Porter's valuable paper on the Pathology of Bone, in Todd's Cyciopadia of Anatomy; and to the section on the osseous system, in Lehmann's Pathological Chemistry.

If I might offer a theory on the state of the bones in the case I have narrated, I should infer that the inequality in the deposition of earthy matters, and the disproportion in construction between the organic and the inorganic parts, was the main predisposing cause of the fractures. In the nodulated parts, there is an increase of earthy deposit; in the constricted parts, the bone was formed, but was too feeble to bear the pressure to which it was subjected. This is the only reasonable sup. position; because, as I have shown, there was an abnormal proportion of organic matter in these bones, taken as a whole.

The connexion or disconnection of the fracrility and abnormal development of bone with the health of the parents, is another matter of prime importance. In this case, I could not detect the slightest hereditary fault. Dr. Montgomery refers to an observation of Jörg's, in which it is argued that the bones of the foetus may be fragile without any special cause.

But, waiving this important point, which can only be fully elucidated by the labours of many inquirers and the lapse of many long days, and proposing a supposition which admits of nodenial, that the bones of this foxtus were in a condition so fragila that the merest force could break them,-we have to consider what force did break them, and when they were broken. From the fact that, in one instance, there was an attempt at reparation, it is clear that some of the fractures occurred long previously to labour. Were the fractures thus produced the result of the fall which the mother experienced? or were they the effect of muscular movements on the part of the child? I would answer this query by saying that, while the fall might, by some inexplicable reason, be the cause of the fractures, the muscular actions natural to the child were quite competent for the purpose; and that, as the fractures seem obviously to have occurred at various times, and as they are in the bones on which the muscles would exert most force, the latter cause is by far the most consistent with all the facts of the case.

Practical Deductrons. From the consideration of the obscure and difficult topics named above, let me now proceed to 
the facts of this case-first, with reference to labour; and secondly, with reference to medico-legal investigations. In these particulars, the case is of direct importance to the working practitioner.

The absence of bone in the superior part of the cranium rendered the nature of the presentation obscure for a short time. Fortunately, I found the membranes presenting in the first instance, and, having ruptured them, was aware that the next structure that met the touch was not the membranous bag containing the amniotic fluid. In a careless practice, or even without carelessness, one might, however, be led to mistake a soft fluid bag of this kind for the membranes, and to rupture it accordingly. Dr. Richardson, indeed, gives a case in point, where, either from a deficiency of bone, or from enlargement of the fontanelle, the tumour presenting was punctured with the finger, and a mass of fluid half-putrid brain-stuff escaped with the liquor amnii. He thus observes in a foot-note in his MS. Fothergillian essay :-

"The case lescribed fixed itself particularly on my mind, from a circumstarice connected with it in the course of the labour. When called to see the woman, the labour pains being violent, I felt the membranes in the vagina very much distended, and I proceeded to rupture them. The thickness of the supposed membranes surprised me; and the fluid that escaped was so thick and purulent looking, that my surprise was increased. After birth, I found that, in penetrating the membranes, I had also penetrated the head through a distended fontanelle, or a deficiently developed osseous covering. As the foetus was and had long been dead in this case, the act was harmless; but it might have been otherwise, and the circumstance furnishes an obstetric lesson worthy of being recorded." in. i (i.)

There are other presentations, with which the one I have described might also be confounded. The breech could scarcely be mistaken for it; at all events, not for any length of time, or after very careful examination. It might, however, be confounded with placental presentation by an inexperienced accoucheur; for the sensation convered to the finger by a head without bones or fontanelles is not altogether unlike that produced by the placental organ. A skilful tactician would detect the difference in the roundness of the tumour formed by the head, in its elasticity, as well as by the connexion which would be traceable between the mass presenting and the orifice of the uterus, to say nothing of the signs of hæmorrhage which would be likely to manifest themselves in the case of placenta prævia, and not to manifest themselves in the other case.

It would be much more difficult to distinguish by vaginal examination a heal without its bones, from a head having upon it a large soft tumour or a hernia cerebri. It might be very difficult to distinguish it at first from a hydrocephalic head where the fontanclles are largely distended. In all these cases, the progress of the labour would reveal the characteristic evidence.

The child I have described died soon after its birth. It had lived in the uterus with its fractured limbs; its viscera were all perfect; and other childien have lived after delivery with frac. tured limbs. Two reasons occur to me why death took place so rapidly; and these reasons are disconnected with the fracture of the long bones. The most likely cause of death is the compression that must have been exerted on the unprotected brain during the parturition. The second is, that, from the condi. tion of the ribs, and the fractured state of the bones into which some of the external chest-muscles are inserted, the act of respiration was never properly established. Whether, had life continued, the bones would have reunited, and the ossifica. tion would have become normal under a new food regimen, is a question only second in difficulty to that which relates to that physiological modification in the nourishment of the fotus in utero, upon which the abnormal construction of the bones depended.

It is not very probable that cases of the description I have given would offer any marked difficulties in the progress of delivery. The non-resistance of the bones would, in fact, be favourable, rather than not, to the parturient act. But a case might occur, in the range of possibility, where the bones, being in a brittle condition, but not broken previously to labour, might, during a severe labour, and during the extraction of a child with instruments, become fractured, and cause the practitioner much anxiety and annoyance. It might, for instance, be urged against him that he had acted unskilfully, or had used too much force, and by so doing had inflicted injuries on the child sufficient to produce death. In such a dilemma the recollection of but one instance resembling the one I have related, will always be satisfactory, and will lead to the careful examination of the bones themselves, ard the discovery of their condition.

But the great practical importance of these cases is to be found in a medico-legal point of view. Here they assume a position which cannot well be over estimated. A child is born; it has been heard to cry, or seen to breathe. The post mortem evidence is in proof of such child baving breathed; the lungs show evidence of having been inflated; the circulatory apparatus gives signs that a free pulmonic circuit has been made. The thoracic and abdominal viscera are all perfect. The muscular structures are well nourished, but the bones are fractured. There are a variety of circumstances which might lead to the supposition that in such a case the mischief had occurred after birth, and had been due to violence intentionally applied. To the best of my knowledge, no such case has occurred; but in the event of the occurrence of an example of the kind, the possibility of intrauterine fracture should always be considered. It should be remembered, moreover, that the child may live a long time after birth, notwithstanding the existence of fracture; during this time modifications in the constitution of the bones might arise, so that the general brittleness of the bones might have become less, and might thus complicate the inquiry. However, with the facts now presented before the mind, it is clearly the duty of every medico-legal inquirer, in the event of injury to the bones of a still-born or living-born child, never to give a syllable of evidence until he has fully examined all the bones of the body; first, in reference to their physical strength; secondly, in regard to the osseous development and the union of bone and cartilage; thirdly, in respect to the presence or absence of certain bones; fourthly, in inquiry as to the existence of evidence, whether there had been fracture and reunion of bone ; fifthly, as to the character of the fracture itself; sixthly, in relation to the presence or absence of external injury; and, seventhly, in relation to the contour of the bones and limbs generaliy. If in any given case it should be detected that the bones were preternaturally brittle,or that there was arrest of osseous development,- - or an absence of bone,- - or sign of preexisting fracture, - or want of traces of external injury,-or, lastly, distinct twist or abnormal contour,in the event of finding any of these peculiarities, I repeat, there would be strong grounds for assuming that the fracture had been intrauterine, and for considering it apart from the idea of the application of any external violence.

Again, a case might perchance arise in which, owing to congenital deficiency, a bone entire at the moment of birth should become fractured after birth from the merest and most unexpected accident. The muscular movements of the child itself, a sudden jerk, the putting on of its dress, a carelessness in nursing, a slight degree of pressure in bed, and so on, might lead to the accident of fracture. In such an example the child might not die, and the possibility of examining the condition of the bone would be, consequently, shut out. Here, therefore, such signs as are purely external would form the chief clue to the true condition of bone; viz., irregular contour, deficient development, and evidence of union by callus in other parts.

Once more, there might be the signs and the fact of fracture, with corresponding signs of external injury. And yet the injury might not stand in the same relation to the fracture as would at first sight be assumed. 'The mark from a fold of cord around the bone, a little extra pressure by the uterus during birth producing ecchymosis, a bruise from a fall, or a tightly fitting and hard article of clothing; these might all attend fracture, and be rather signs of secondary causes, or of coincidences, than veritable and efficient causes of the mischief.

Conclusron. I cannot conclude this paper without urging on my brethren engaged in obstetric practice, the fact, that they cannot be lending more valuable assistance to science than when they are engaged in tracing out the existence of fotal diseases, the tranmission of such diseases from the parent to the cffspring, the original development of diseased conditions in utero as depending on modifications of the placenta and the uterine appendages, and the influence of diseases contracted in intrauterine life on the health of the future woman or man. The subjects here inrolved are amongst the profoundest in medical science, and they lie the most freely open for inspection. We have not to go out of the path of our ordinary practical duties to seek for them, but we cannot perform the duties incident to practice without meeting with them perforce. Every still-born child presents a field for pathological inquiry; but for the dissector's knife to touch a still-born child is an exceptional fact. I regret myself having, on occasion after 
occasion, let the opportunity go by for advancing uterine pathology, as though it were of no moment; and I must not, consequently, blame others, but rather try to improve myself, and ask my friends to move with me. As yet, the literature of medicine has not produced more than sixty-six contributions on fœtal diseases, and the majority of these are only stray, and often imperfectly reported cases. How important, then, that all cases of fotal disease should be frorn henceforth recorded, that some central mind may group such diseases together in their respective classes, and elaborate a true and philosophical system of uterine pathology.

P.S.-It may be worthy of remark that the lady, Mrs. L., who in September, 1856 , gave birth to the foetus, the subject of the foregoing remarks, was yesterday (September 2ith) delivered of a healthy and well formed female child at the full period.

Bedford, September 28 th, 1857

\section{THE PHYSIOLOGY, PATHOLOGY, AND THERA- PEUTICS OF THE MOTOR FUNCTIONS OF THE UTERUS.}

By H. Hannotte Vernon, M.D., Physician to the Great Northern Hospital; formerly Resident-Accoucheur to St. Mary's Hospital.

[Continued from page 642.]

Part II.-Pathology (concluded).

The next nervous centre which arrests attention is-

3. The Spinal Corl. The general effect of the exaggerated importance which has, I believe, been attached to the spinal cord, as far as the physiology of labour is concerned, has been to exalt also its pathological importance. There are doubtless many and very grave circumstances which arise ont of morbid states of the spinal medulla, whether these consist of conditions existing in the tissues of the cord, or from states of the economy at large. But what appears to me necessary in relation to the influence of the spinal cord on morbid labour is, to divest the professional mind of the idea that all or nearly all aberrations of excito-motor action of the uterus are aberrations of spinal innervation. The anatomical fact of the extremely scanty supply of spinal nerves to the uterus itself, is evidence enough that the primary derangements of uterine nervi-motor action must spring from another source. What that source is $I$ have been at some pains to show. The general physiological law as to the readiness of conduction or diffusion of impressions from one nervous centre to another, being in direct proportion to their intensity, is, moreover, another light by which to read the pathological importance of the spinal cord. The additional fact that organs in the immediate vicinity of the uterus, or in close sympathy with it, are (with the exception of the ovaries) well supplied with spinal nerves, points out that whatever influence the cord exerts upon labour must depend chiefly upon two things; first, upon excitations of other organs than the uterus, of considerable intensity; or, secondly, upon very marked morbid conditions of the spinal cord itself. The locality of eccentric excitations has been discussed before, and attention must nowitherefore be confined to conditions of the medulla itself. The period of labour at which morbid states of the spinal cord produce their effect upon the parturient act is when dilatation of the os uteri has been accomplished, or nearly so, and when the presenting part of the fortus has begun to impinge upon and excite those organs and muscles which are supplied with spinal nerves. The exceptions as to the period at which the state of the cord begins to influence labour, arise out of the intensity of the morbid states; for it may be easily seen that extreme polarity of the centre in question will be diffused and conducted to the ganglia of the uterus proper, and thus modify the peristaltic or primary movements of labour from the very beginning. Morbid states of a minor degree do not declare themselves until the nerves of the cord itself are implicated, or until the voluntary and concomitant consensual movements of labour should exhibit themselves.

It is only necessary, in regard to the spinal cord, to resolve its various morbid modes of action into two conditions, which underlie them all ; viz., $(a)$ hyperæsthesia, and $(b)$ hypæsthesia. These two ideas are simple enough, and under them I believe we can range all aberrations of nerve action in relation to uterine motor action. Protean as are the secondary causes of spasm on the one hand, or inertia on the other, they all, tith the exception of structural changes in the uterus itself, end in exalted and duminished polarity of nervous centres, topical, or general, or induced by eccentric irritations.

a. Hyperæsthesia of the cord results in the first place from conditions of other parts of the nervous system. Emotions and energetic cerebration would appear in certain instances to throw the spinal cord into a state of undue polarity. When this is the case, the most trifling eccentric irritations will suffice to set up violent reflex actions. The first pains of labour, instead of remaining dependent almost entirely upon ganglionic action, are speedily linked with spinal action. Irritations arising in the uterus itself, which, under ordinary circumstances, would not have been diffused so far, are felt by the cord; and the actions normally peculiar to a more advanced stage of labour are provoked almost at the onset. It is thus that we meet, from time to time, with cases in which, whilst the os uteri is almost closed, there are strenuous efforts, not entirely, though partly, voluntary. Such cases are not instances of mere perversity of the patient; she may close her glottis, depress her diaphragm, and call into action all the muscular power she possesses at a time when such efforts can be of little avail ; but the voluntary effort is only auxiliary to a more powerful spinal impulse, which would produce a part of the effort quite independently of volition. It is curious to observe how different this confused, transposed, and misplaced action of the nervous centres is from normal action. When labour progresses under strictly physiological conditions, the first step in the process is to open the mouth of the uterus; for this purpose the uterus suffices for itself, its own muscles, impelled by its own nervous centres, are allsufficient. No imaginable action of the diaphragm and abdominal muscles can affect the opening of the os uteri until it is very far advanced in dilatation; and hence we find that normally the spinal cord and the voluntary muscles are not called isto play, until it is possible for them to produce their proper effect. This is a beautiful instance of the economy of nature in producing results. As soon as volition is efficient, it is utilised, and no sooner; and so with reflex action also.

Hyperasthetic conditions of the uterine ganglia, and of nerveterminations in all parts of the body, sometimes the result of perverted vascular actions in the tissues in which the nerveterminations lie, are not unfrequent causes of undue polarity of the cord. This idea I am anxious to impress upon the minds of my readers; viz., that, owing to the close intercommunication between all parts of the nervous system, persistent or intense peripherical irritations, or centric excitalility of any one centre, invariably results in exalted polarity of all nervous centres. This is a fundamental fact, which it is necessary to bear in mind as explanatory of numerous disorders of innervation, observable not merely in the department of obstetric pathology, but in pathology at large.

The general relation of spinal polarity to polarity of other ganglia or systems of ganglia having been indicated, it is quite unnecessary to go into mere anatomical details. These are readily supplied by the most ordinary anatomical knowledge.

Another form of spinal hyperæsthesia which must be familiar to every obstetrician is that which is at once idiopathic and idiosyncratic; that is to say, peculiar to the individual, and traceable to no anterior pathological condition. It is impossible to offer any further explanation of such a condition than one which shall resolve itself into a merely hypothetical mode of expressing the fact. Why certain individuals should generate a more abundant supply of nerve-impulses than others, is wholly unaccountable. We may hypothecate a greater proportion of grey matter, or more rapid molecular changes, or twenty other imaginary conditions or modes of action; but the thing still remains really unexplained. It appears to be an ultimate fact, that persons of different temperaments possess a vitality which manifests itself in different parts of their organisation. Some affect great vegetative tendencies, and grow into human mountains upon what others would almost starve upon. Others possess a vivacity of nervous constitution, which shows itself in rapid cerebration, rapid and agile adaptive movements, and susceptibility to all kinds of impressions, whether physical or emotional. It transcends the knowledge of the physiologist to say upon what this depends; the scalpel and the microscope are useless means of investigation here, and we are reluctantly compelled to suspend inquiry just where inquiry is the most inviting.

Certain kinds of toxæmia appear to throw not only the spinal cord but all nervous centres into a high state of excitability. 